\title{
Toward Application of Virtual Reality to Visualization of DT-MRI Volumes
}

\author{
S. Zhang ${ }^{1}$, Ç. Demiralp ${ }^{1}$, M. DaSilva ${ }^{1}$, D. Keefe ${ }^{1}$, D. Laidlaw ${ }^{1}$, \\ B.D. Greenberg ${ }^{1}$, P.J. Basser ${ }^{2}$, C. Pierpaoli ${ }^{2}$, E.A. Chiocca ${ }^{3}$, and \\ T.S. Deisboeck ${ }^{3}$ \\ 1 Brown University, Providence, RI 02912, USA \\ 2 STBB, NICHD, National Institutes of Health, Bethesda, MD 20892, USA \\ 3 Neurosurgical Service and Brain Tumor Center, Mass. General Hospital, Harvard \\ Medical School, Boston, MA 02114, USA
}

\begin{abstract}
We describe a virtual reality application for visualizing tensorvalued volume data acquired with diffusion tensor magnetic resonance imaging (DT-MRI). We have prototyped a virtual environment that displays geometric representations of the volumetric 2nd-order diffusion tensor data and are developing interaction and visualization techniques for two application areas: studying changes in white-matter structures after gamma-knife capsulotomy and pre-operative planning for brain tumor surgery.
\end{abstract}

\section{Introduction}

Diffusion Tensor Magnetic Resonance Imaging (DT-MRI) has the potential to measure fiber-tract trajectories in soft fibrous tissues, such as nerves, muscles, ligaments, and tendons [1]. However, the datasets produced are volumetric with six values at each spatial location and, hence, present a significant challenge to visualize and understand. The potential for these multivalued volume images is likely to be great but is, as yet, not well explored.

A number of methods have been developed that display the data as $3 \mathrm{D}$ models 23. Some methods, including ours, track the fiber-tract trajectories in DT-MRIs 124 . We generate a large set of streamtubes and streamsurfaces to represent, respectively, linear structures like fiber tracts and planar structures. With traditional monocular single-screen displays, it is difficult to interpret these complex geometric models. The situation is exacerbated when the complexity of the models is increased to meet user requirements for more detail. An immersive virtual environment such as the Cave [5] has the advantages of head-tracked stereo display, a large display surface, and interactivity [10. For some tasks, users can improve performance on similar complex models in such an environment by $200 \%$ over working with a static image 9]. Virtual environments have already been used in some surgical applications [11].

This short paper describes our efforts to use the Cave for DT-MRI visualization. Our targeted applications include studying changes in white matter structures before and after gamma-knife capsulotomy and pre-operative planning for brain tumor surgery. 


\section{Method}

Virtual Reality Setup Our Cave is an $8 \times 8 \times 8$ foot cube with rear-projected front and side walls and a front-projected floor. A user wears a pair of LCD shutter glasses that support stereo viewing. The glasses have an attached tracker that relays their position and orientation to the computer. In our application, the virtual environment consists of a room the size of the Cave itself and a table under the visualization of the DT-MRI data. The user can walk around, bend over, and move his/her head to observe the visualization from different perspectives.

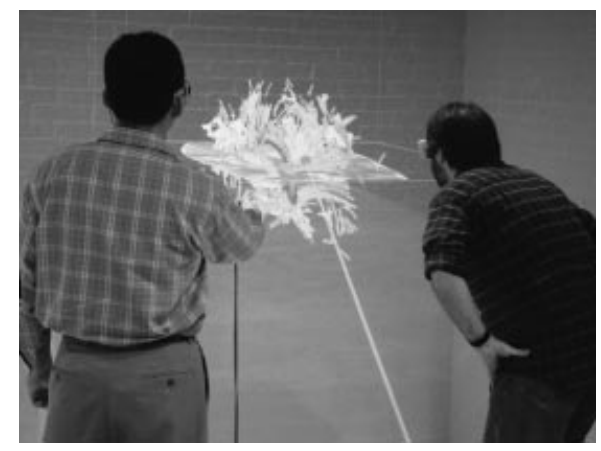

Fig. 1. Two users observe the DT-MRI Fig. 2. A user takes a closer look by visualization in the Cave.

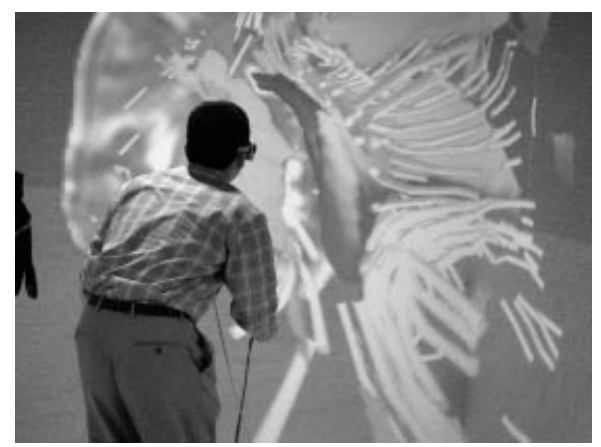

moving toward the virtual brain.

DT-MRI Visualization We have displayed DT-MRI and T2-weighted images of a human brain (courtesy Dr. Susumu Mori, Johns Hopkins University). Both of the datasets are registered in a $256 \times 256 \times 40$ volume. Each voxel is $0.89 \times$ $0.89 \times 3.2 \mathrm{~mm}$.

We use three kinds of geometric models, streamtubes, streamsurfaces, and isosurfaces, to present different types of structures in the brain. Fibrous structures in the brain, like white-matter tracts, yield diffusion tensors with linear anisotropy. Streamtubes are thin tubes that follow the direction of fastest diffusion in regions of linear anisotropy. Analogously, streamsurfaces follow planar structures in regions of planar anisotropy. Colors are mapped on the geometric models to display the magnitude of linear or planar anisotropy. Initially we generate the geometric models from a dense set of seed points in the volume. We then use a culling algorithm to pick a representative subset for display [46]. We also generated isosurfaces of the ventricles from the T2-weighted image to give the user an anatomical context.

The doctors who first used our system suggested that viewing typical $2 \mathrm{D}$ T2-weighted sections might help in identifying anatomical features in the 3D models. We provide a $2 \mathrm{D}$ slicer that the user can move through a 3D scalar dataset that is co-registered with the DT-MRI data. The user can also change the dataset carried by the slicer. We have displayed the T2-weighted image with 
the slicer to validate the ventricle models, displayed the linear anisotropy image to validate and understand the streamtube models, and displayed the planar anisotropy image to validate and understand the streamsurface models.

Interaction The current interaction scheme is a simple one. The geometric models and sections appear stationary above the virtual table. The user walks around and changes head position to examine the data from different perspectives. With a three-button wand, the user can point to features of interest using a virtual laser pointer, can change the position of the slicer with two of the buttons, or can choose among axial, coronal, or sagittal sections with the third button.

\section{Discussion and Conclusion}

We have constructed a virtual environment for visualizing tensor-valued volume data acquired with DT-MRI.

Three of the authors who are MDs have used the system and provided feedback. Two are neurosurgeons from MGH and one an MD/PhD studying obsessive compulsive disorder at Butler Hospital in Providence, RI. Their experience with the system suggests that it is sufficiently easy to use. They had each also viewed static images of the geometric models and found it easier to visualize the 3D structures in the virtual environment. The $2 \mathrm{D}$ sections were reported to be a very valuable additional tool within the virtual environment.

Our long-term goal is to provide an efficient and effective visualization of DT-MRI volume data together with other medical imaging modalities. As effectiveness can be measured only in the context of a specific application, we intend to pursue the applications we have described. As a first step, we have constructed a framework in which geometric models are put into a fully immersive virtual environment. Users move in the virtual environment and observe the visualization in the same way they would view a physical object in the real world. The environment shows strong potential for understanding these complicated datasets and the underlying anatomy and pathology that they measure.

\section{References}

1. Peter Basser et al., Magn. Reson. Med., 44:625-632 (2000)

2. Rong Xue et al., Magn. Reson. Med., 42:1123-1127 (1999)

3. Gordon Kindlmann et al., Proc. IEEE Visualization, 183-189 (1999)

4. Song Zhang et al., Proc. ISMRM (2001)

5. Carolina Cruz-Neira et al., SIGGRAPH, 135-142 (1993)

6. Song Zhang, Master's Thesis, Brown University (2000)

7. Will Schroeder et al., The Visualization Toolkit, Prentice Hall (1996)

8. Enrico Gobetti et al., Proc. IEEE Visualization, 435-438 (1998)

9. Colin Ware et al., ACM Transactions on Graphics, 15(2)121-140 (1996)

10. Andrew Forsberg et al., Proc. IEEE Visualization, 457-460 (2000)

11. S. Taylor et al., Computer-Integrated Surgery, MIT Press (1996) 